\title{
Zur Sprache gebracht
}

\section{Carlos B. Quinto}

Dr. med., Mitglied des FMH-Zentralvorstandes, Departementsverantwortlicher Public Health und Gesundheitsberufe

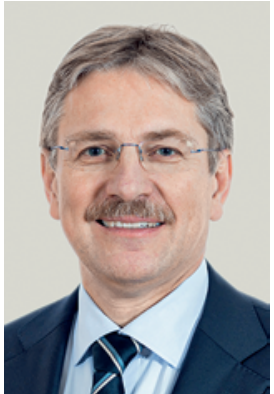

Wir befinden uns in einer Welt, in der die unterschiedlichsten Sprachen und Kulturen aufeinandertreffen und miteinander koexistieren. Besonders in der Schweiz mit vier offiziellen Landessprachen und einem hohen Anteil ausländischer Einwohner ist die Vielfalt der Sprachen allgegenwärtig. In ihrer täglichen Arbeit sind Sie, liebe Mitglieder, auf die sprachliche Verständigung mit Ihren Patientinnen und Patienten angewiesen. Die Erhebung einer vollständigen Anamnese, die Verordnung weiterer Untersuchungen oder Therapien, Dosierungs- und Einnahmeangaben zu Medikamenten, die Adhärenz zur gewählten Behandlung und die gemeinsame Entscheidungsfindung zeigen nur

\section{In ihrer täglichen Arbeit sind Ärztinnen und} Ärzte auf die sprachliche Verständigung mit ihren Patientinnen und Patienten angewiesen.

einen Bruchteil der kommunikativen Herausforderungen auf. Bereits in der Ausgabe vom 3. Februar 2021 erschien ein Beitrag, der das Missverhältnis zwischen Nachfrage und Bereitstellung von Dolmetscherleistungen aufzeigt.

Oft treten die Angehörigen der Patienten und Patientinnen als Vermittler und Übersetzer in Situationen sprachinkongruenter Konsultationen auf. Ob Laien die Fähigkeit besitzen, adäquat zu vermitteln, und ob sie das volle Vertrauen der Patienten und Patientinnen erhalten, um frei sprechen zu können, ist fraglich. Tabuthemen können den Informationsfluss entscheidend verringern, zum vermeintlichen Schutz der Betroffenen können Informationen vorenthalten werden.

Professionelle interkulturelle Dolmetschende überwinden die Barrieren in der schwierigen Kommunikation zwischen Arzt und Patient: freie Äusserung von Beschwerden und Meinungen sowie die Anpassung an kulturelle Unterschiede sind nur wenige davon. Einen einfachen und benutzerfreundlichen Zugang zu Dolmetscherleistungen vorausgesetzt, könnten Ärztinnen und Ärzte in sprachlich herausfordernden Situationen die Patienten und Patientinnen optimal versorgen.

Hierfür müsste die Organisation von Dolmetscherleistungen wesentlich vereinfacht werden, und ihre
Finanzierung müsste einfach sichergestellt werden. Während im TARMED keine Positionen für das Dolmetschen enthalten sind, könnten solche im TARDOC in gemeinsamen Verhandlungen zwischen den Tarifpartnern eingeführt werden. Nur müsste TARDOC endlich vom Bundesrat genehmigt werden.

Ebenfalls müssten die aktuellen Zeitlimitationen in dieser Hinsicht korrigiert werden. Krisen und somit die Konsultationen in Hausarztpraxen verlaufen nicht linear übers Jahr verteilt. Ungenügende oder mangelhafte Kommunikation führt nachweislich zu mehr Untersuchungen und Tests sowie auch zu mehr Fehldiagnosen. Nicht vorhandene Gesprächszeit aufgrund des bundesrätlichen Tarifeingriffs und nicht vorhandene Dolmetscherleistungen führen somit zu einer teureren und schlechteren Medizin, für die nicht die Ärzteschaft in der Verantwortung steht. Dies konnte im Inund Ausland durch Studien belegt werden, die unter anderem bereits 2016 an einer Swiss Public Health Conference, an der sogar ein Bundesrat anwesend war, in Bern präsentiert wurden.

Es gibt Verbesserungsbedarf. Dies wurde für eine vulnerable Untergruppe der Migrationsbevölkerung am 8. Dezember 2020 deutlich ausgeführt an einer Fachtagung des Schweizerischen Roten Kreuzes des Verbundes Support for Torture Victims mit dem Titel «Lost in Translation - interkulturelles Dolmetschen in der Gesundheitsversorgung geflüchteter Menschen»' In den Unterlagen zur Fachtagung finden Sie entspre-

\section{Professionelle interkulturelle Dolmetschende} überwinden die Barrieren in der schwierigen Kommunikation zwischen Arzt und Patient.

chende weiterführende Literatur und Präsentationen. In der abschliessenden Diskussion zur Tagung zeigte sich, dass die Probleme nicht bei den dort anwesenden lösungsorientierten Vertretern von Ärzteschaft und Versicherern lagen, sondern in den fehlenden Strukturen, um finanzierte Dolmetscherleistungen rasch und unkompliziert zu beziehen.

1 www.torturevictims.ch/de/veranstaltungen/lost-in-translationinterkulturelles-dolmetschen-in-der-gesundheitsversorgung 